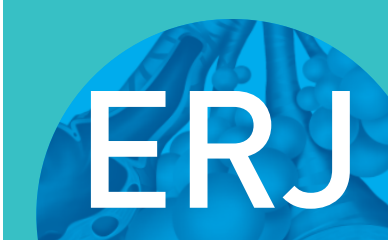

open research

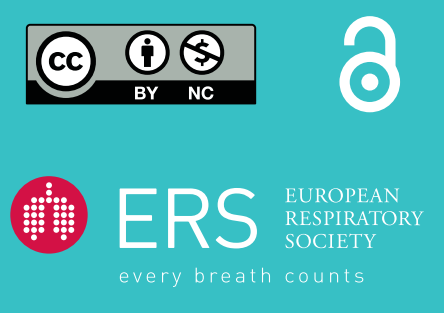

\section{Newly developed multiple-breath washout reference equations from the CHILD Cohort Study: implications of poorly fitting equations}

\section{To the Editor:}

The multiple-breath washout (MBW) test allows for the measurement of lung volumes, ventilation homogeneity and gas clearance curves during tidal breathing [1]. Lung clearance index (LCI), the most commonly reported parameter from the MBW test, is a marker of ventilation inhomogeneity and is associated with early lung disease. LCI is defined as the cumulative expired volume divided by the functional residual capacity (FRC). The currently accepted reference equation for LCI measured using sulfur hexafluoride $\left(\mathrm{SF}_{6}\right)$ shows a non-linear decrease as age and height increase, with height as an independent predictor from infancy to school-age [2]. In contrast, FRC shows a non-linear increase in FRC as age and height increase with males having higher measured FRC compared to female subjects.

Reference data based on healthy subjects may vary due to differences in population characteristics, testing equipment and software [3]. Inappropriate selection of reference values will provide incorrect estimates of an individual's or group's z-score, resulting in misinterpretation of results [4]. Therefore, appropriate representative normative reference data must be available to correctly interpret lung function results. This is often challenging for infant and paediatric datasets, which tend to be smaller in scale than in older children and adults. In this study, we developed a set of new reference equations for $\mathrm{SF}_{6} \mathrm{MBW}$ parameters based on data collected from the Canadian Healthy Infant Longitudinal Development (CHILD) Cohort Study, a large Canadian general population multi-ethnic birth cohort study [5]. Our newly developed reference equations were compared with the published reference equations from the 2013 study of LuM et al. [2]. In addition, we evaluated pulmonary function z-score values generated by these two equations in children recruited locally from our asthma clinic with recurrent wheezing.

For our reference equation, healthy children aged 3-60 months enrolled in the Toronto site of the CHILD Study were recruited for this study. Children who demonstrated prior respiratory distress, had recurrent wheezing at the time of tests or were exposed to maternal smoking during pregnancy were excluded. MBW was performed in infants under 6 months during natural quiet sleep, and during quiet sleep after sedation in infants older than 6 months to 2 years, and finally while awake and sitting upright at their 3 and 5 years clinic visits. MBW measures were performed using a mass spectrometer (AMIS 2000; Innovision $\mathrm{A} / \mathrm{S}$, Odense, Denmark) with a gas mixture of $4 \% \mathrm{SF}_{6}$ as a marker gas as previously described [6]. All MBW data met the American Thoracic Society/European Respiratory Society (ATS/ERS) quality control standards $[7,8]$. To account for repeated measures within subjects, we applied general mixed effect models with random intercepts for investigating the best fit model for each lung function outcome. Reference equations were then constructed using the LMS (lambda-mu-sigma) method. The LMS method allows for modelling the skewness (L), median (M) and coefficient of variation (S) simultaneously [9]. Then the $\mathrm{z}$-score can be calculated using the formula: $\mathrm{z}$-score $=\left((\text { measurement } / \mathrm{M})^{\mathrm{L}}-1\right) /(\mathrm{L} \times \mathrm{S})$

@ERSpublications

Using inappropriate reference equations would provide incorrect estimate of $\mathrm{z}$-scores, which would cause misdiagnosis. Appropriate representative normative reference data must be available to correctly interpret individual lung function results. https://bit.ly/3dcNZ5p

Cite this article as: Lu Z, Dai R, Kowalik K, et al. Newly developed multiple-breath washout reference equations from the CHILD Cohort Study: implications of poorly fitting equations. ERJ Open Res 2021; 7: 00301-2020 [https://doi.org/10.1183/23120541.00301-2020]. 
We included 280 healthy subjects with 644 test occasions in the current analysis. There were 261 test occasions from infancy, 186 from 3 years and 197 from 5 years. Of these 280 subjects, 71 (25\%) provided data on one test occasion, $105(38 \%)$ on two test occasions, $59(21 \%)$ on three test occasions and $45(16 \%)$ on four or five test occasions. There were 149 (53\%) males and 155 (55\%) were Caucasian. The mean (standard deviation, SD) age was 35 (21) months. The weight-for-age $\mathrm{z}$-score and height for age $\mathrm{z}$-score according to the World Health Organization (WHO) standard [10] were 0.15 (0.93) and -0.11 (1.01), respectively. Our infant and preschool population were similar to the height and weight of those in the Lum et al. study, although our study had a smaller proportion of Caucasians $(\mathrm{p}<0.001)$.

In the univariate model, age, height, weight and sex were associated with LCI values. In the multivariate model, we found that both age and height remained significant determinants for LCI. Specifically, LCI decreased nonlinearly as age and height increased. For FRC, age, height and sex were significant factors in the multivariate model while ethnicity was not. Our newly developed reference equations for both LCI and FRC (figure 1a) provided a good fit for our healthy subjects and thus, gave z-scores with mean 0 and SD 1, respectively. The original Lum et al. z-scores were solely dependent on height, and when applied to the CHILD data, we noted the LCI was significantly reduced by a mean (SD) z-score of $-1.15(1.21, \mathrm{p}<0.001)$, with an overdistribution of values below the lower limit of normal ( $-1.64 \mathrm{z}$-score). Notably, the proportion of children falling below the lower limit of normal increased with the age of the subjects. The offset between the developed z-scores and Lum et al. z-scores began from infancy and became much worse at 3 and 5 years (figure 1b). The noted differences in LCI between our study and the LuM et al. study remained even when the analysis was restricted to Caucasian children. Although Toronto data were included in the original Lum reference equation, data from our centre were limited to infancy due to the age of our cohort at the time of the previous publication. As a result, when we limited our analysis to infant ages, differences between equations are less striking than reported in the original publication. Notably, the original Lum equation for FRC z-scores fit our population well (figure 1c) despite the offset in the LCI z-scores. We did note that the respiratory rate (RR), however, was on average 0.9 unit lower $(\mathrm{p}=0.02)$ in the CHILD Cohort Study, after adjustment for age and height, which may partially explain the difference in LCI between our study and the Lum et al. study. The reasons for the lowered RR are not clear; both the CHILD and Lum studies used sedation for infant studies and videos to distract preschool children studied with a face mask while sitting upright.

Next, we assessed the clinical impact of the use of our novel reference equation on interpretation of LCI data from clinical groups. MBW data from 37 children diagnosed with recurrent wheeze have previously been reported from our centre [11]. These patients had a mean (range) age of 1.6 (0.62 to 2.93) years, 25 (68\%) were male and 21 (57\%) were Caucasian. Use of the LuM et al. reference equations produced a mean (SD) LCI $\mathrm{z}$-score of 0.26 (1.61) for this group of symptomatic infants, which falls within the range for healthy children $(\mathrm{p}=0.34)$. In contrast, when our newly developed equations were used, the mean (SD) LCI $\mathrm{z}$-score was 0.99 (1.65), which is significantly increased (worse) compared to the general population $(\mathrm{p}<0.001)$. Similarly, using an upper limit of normal (1.64 z-score) as a cut-off, the Lum et al. equation identified $19 \%$ of these children as having abnormal LCI, whereas our developed reference equations identified $35 \%$ of these wheezing infants as having abnormal LCI. Therefore, the use of the Lum equation would have led to misclassification of pulmonary function in children with a disease as "normal" nearly half of the time.

a)

\begin{tabular}{|c|c|}
\hline MBW parameters & Developed reference equations ${ }^{\#}$ \\
\hline \multicolumn{2}{|l|}{$\mathrm{LCl}$} \\
\hline M & $\begin{array}{c}1.8606-1.0399 \cdot \text { age }+0.1210 \cdot \text { age }^{2}+ \\
12.5766 \cdot \text { height }-6.3428 \cdot \text { height }^{2}\end{array}$ \\
\hline S & 0.077 \\
\hline $\mathrm{L}$ & -0.5565 \\
\hline \multicolumn{2}{|l|}{ FRC L } \\
\hline M & $\begin{array}{l}0.8238+0.0740 \cdot \text { age }-0.0068 \cdot \text { age }^{2}- \\
2.1672 \cdot \text { height }+1.6568 \cdot \text { height }^{2} \\
0.0075 \cdot \text { sex }\end{array}$ \\
\hline S & 0.1615 \\
\hline $\mathrm{L}$ & 0.2597 \\
\hline
\end{tabular}

b)

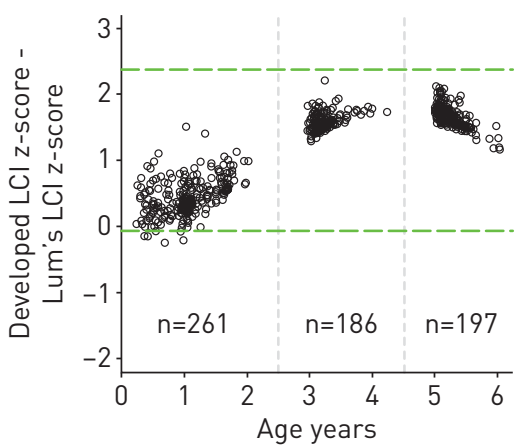

c)

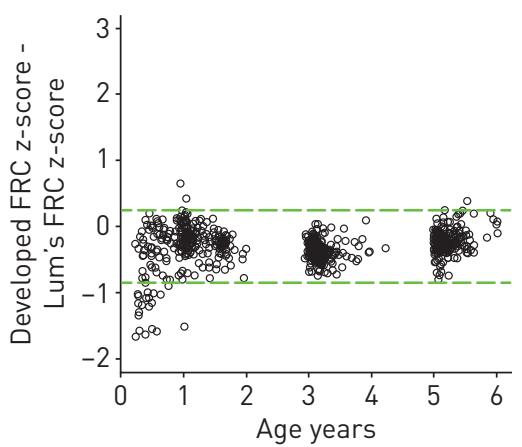

FIGURE 1 a) Newly developed reference equations for multiple-breath washout (MBW) data from the CHILD study. b) Comparison between developed reference equations and LUM et al. [2] reference equations for lung clearance index (LCI) in CHILD study subjects. C) Comparison between developed reference equations and Lum et al. [2] reference equations for functional residual capacity (FRC) in CHILD study subjects. Dashed lines denote the $95 \%$ confidence interval of the difference calculated as developed z-score - Lum's z-score. M: predicted median; $S$ : coefficient of variation; L: skewness." : age in years, height in metres, and sex=1 for male, 0 for female). 
In conclusion, we found that LCI is dependent on both age and height rather than height alone. Our newly developed reference equations provide normative data for the CHILD Cohort Study and other centres with similar population characteristics that utilise this equipment.

Zihang Lu ${ }^{1,2}$, Ruixue Dai ${ }^{1}$, Krzysztof Kowalik ${ }^{1}$, Aimee Dubeau ${ }^{1}$, Diana L. Lefebvre ${ }^{3}$, Susan Balkovec ${ }^{1}$, Allan B. Becker ${ }^{4}$, Piush J. Mandhane ${ }^{5}$, Stuart E. Turvey $\oplus^{6}$, Wendy Lou ${ }^{7}$, Felix Ratjen ${ }^{1}$, Theo J. Moraes ${ }^{1}$, Malcolm R. Sears ${ }^{3}$, Per M. Gustafsson ${ }^{8}$ and Padmaja Subbarao ${ }^{1,7}$

${ }^{1}$ Division of Respiratory Medicine, Dept of Pediatrics and Program in Translational Medicine, SickKids Research Institute, The Hospital for Sick Children, and University of Toronto, Toronto, Canada. ${ }^{2}$ Dept of Public Health Sciences, Queen's University, Kingston, Canada. ${ }^{3}$ Dept of Medicine, McMaster University, Hamilton, Canada. ${ }^{4}$ Dept of Pediatrics and Child Health, University of Manitoba, Children's Hospital Research Institute of Manitoba, Winnipeg, Canada. ${ }^{5}$ Dept of Pediatrics, University of Alberta, Edmonton, Canada. ${ }^{6}$ Dept of Pediatrics, Child and Family Research Institute, BC Children's Hospital, University of British Columbia, Vancouver, Canada. ${ }^{7}$ Dalla Lana School of Public Health, University of Toronto, Toronto, Canada. ${ }^{8}$ Dept of Pediatrics, Central Hospital, Skövde, Sweden.

Correspondence: Padmaja Subbarao, The Hospital for Sick Children, Peter Gilgan Center for Research and Learning, 686 Bay Street, Toronto, Ontario M5G 0A4, Canada. E-mail: padmaja.subbarao@sickkids.ca

Received: 24 May 2020 | Accepted after revision: 2 Oct 2020

Conflict of interest: Z. Lu has nothing to disclose. R. Dai has nothing to disclose. K. Kowalik has nothing to disclose. A. Dubeau has nothing to disclose. D.L. Lefebvre has nothing to disclose. S. Balkovec has nothing to disclose A.B. Becker has nothing to disclose. P.J. Mandhane has nothing to disclose. S.E. Turvey has nothing to disclose. W. Lou has nothing to disclose. F. Ratjen reports grants and personal fees from Vertex, and personal fees from Novartis, Bayer, Roche and Genetech, outside the submitted work. T.J. Moraes has nothing to disclose. M.R. Sears has nothing to disclose. P.M. Gustafsson has nothing to disclose. P. Subbarao has nothing to disclose.

Support statement: This study was supported by the CIHR (grant 48616), AllerGen NCE (project 07A1.2), Don and Debbie Morrison, and the SickKids Foundation. Funding information for this article has been deposited with the Crossref Funder Registry.

\section{References}

1 Subbarao P, Milla C, Aurora P, et al. Multiple-breath washout as a lung function test in cystic fibrosis. A Cystic Fibrosis Foundation Workshop Report. Ann Am Thorac Soc 2015; 12: 932-939.

2 Lum S, Stocks J, Stanojevic S, et al. Age and height dependence of lung clearance index and functional residual capacity. Eur Respir J 2013; 41: 1371-1377.

$3 \mathrm{Lu} \mathrm{Z}$, Foong RE, Kowalik K, et al. Reference equations for the interpretation of forced expiratory and plethysmographic measurements in infants. Pediatr Pulmonol 2018; 53: 907-916.

4 Lum S, Bountziouka V, Wade A, et al. New reference ranges for interpreting forced expiratory manoeuvres in infants and implications for clinical interpretation: a multicentre collaboration. Thorax 2016; 71: 276-283.

5 Subbarao P, Anand SS, Becker AB, et al. The Canadian Healthy Infant Longitudinal Development (CHILD) Study: examining developmental origins of allergy and asthma. Thorax 2015; 70: 998-1000.

6 Gustafsson P, Aurora P, Lindblad A. Evaluation of ventilation maldistribution as an early indicator of lung disease in children with cystic fibrosis. Eur Respir J 2003; 22: 972-979.

7 Robinson PD, Latzin P, Ramsey KA, et al. Preschool multiple-breath washout testing. An official American Thoracic Society technical statement. Am J Respir Crit Care Med 2018; 197: e1-e19.

8 Robinson PD, Latzin P, Verbanck S, et al. Consensus statement for inert gas washout measurement using multiple-and single-breath tests. Eur Respir Soc 2013; 41: 507-522.

9 Cole T, Stanojevic S, Stocks J, et al. Age-and size-related reference ranges: a case study of spirometry through childhood and adulthood. Stat Med 2009; 28: 880-898.

10 World Health Organization. WHO Child Growth Standards: Length/Height-for-Age, Weight-for-Age, Weight-for-Length, Weight-for-Height and Body Mass Index-for-Age: Methods and Development. Geneva, World Health Organization, 2006.

$11 \mathrm{Lu} Z$, Foong RE, Kowalik K, et al. Ventilation inhomogeneity in infants with recurrent wheezing. Thorax 2018; 73: 936-941. 